\title{
Análisis de procesos con simulación dinámica como método de aprendizaje en ingeniería
}

DOI: $10.46932 / \mathrm{sfjdv} 2 \mathrm{n} 1-071$

Received in: November 1st, 2020

Accepted in: December 30th, 2020

\author{
Dr. Jesús Vicente González Sosa \\ Ing. Diana Laura Jiménez Díaz \\ Universidad Autónoma Metropolitana, Unidad Azcapotzalco. \\ Departamento de Sistemas, CBI. \\ Av. San Pablo 180, Col. Reynosa Tamaulipas, \\ Delegación Azcapotzalco, C.P. 02200, Cd. México. \\ E-mail: jvgs@azc.uam.mx
}

\begin{abstract}
RESUMEN
La simulación como parte de la preparación de un profesionista se ha convertido en una de las áreas de desarrollo fundamental para la sociedad educativa, dado el impacto que tiene en el desarrollo académico, por ello se debe generar de manera constante una mejora en la implementación de métodos enseñanzaaprendizaje referidas a este rubro, ante esta situación día a día se generan opciones para agilizar y comprender a detalle las temáticas de la ingeniería, a través de simulaciones dinámicas, las cuales ofrecen perspectivas tangibles, desde un punto de vista virtual, de los fenómenos a estudiar y analizar en las diferentes áreas de la ingeniería.

En relación con ello se han generado métodos por medio de diferentes herramientas dinámicas para lograr analizar procesos enfocados en la ingeniería, utilizando software que permite elaborar diagramas, algoritmos y procesos con sus respectivos parámetros de control, con lo cual se enriquece el aprendizaje de los estudiantes cuando logran apreciar fenómenos teóricos en casos prácticos y con alternativas de solución viables de acuerdo con la simulación dinámica obtenida a través del software.

Como parte de los resultados se tienen casos de estudio identificados y mejorados con el uso de la herramienta dinámica, pretendiendo generar una base de casos para el aprendizaje de la ingeniería, ubicándolos en la nube y asimismo aprovechar las tecnologías actuales, enfatizando que se hace uso de las herramientas para lograr una mejora en la enseñanza-aprendizaje.
\end{abstract}

Palabras clave: aprendizaje, metodología de enseñanza, herramienta virtual didáctica, simulación, casos de estudio.

\section{INTRODUCCIÓN}

La dinámica de sistemas en la aplicación de la ingeniería tiene un impacto interesante dada la aplicación, ya que integra diversas disciplinas tecnológicas, teóricas y de ciencia para abordar problemáticas de toda índole, desde la parte social hasta la innovación, cuyo propósito radica en fomentar nuevas tendencias en la enseñanza de la ingeniería fortaleciendo en todo momento las herramientas del docente para su desarrollo dentro del aula (Martínez, 2012). La aplicación de los modelos dentro de la dinámica de sistemas sirve como apoyo, gráfico y visual, para el desarrollo del conocimiento en el área que se aplique, con el propósito de mejorar los procesos de enseñanza. 
En la aplicación la dinámica de sistemas incentiva al análisis de cualquier caso o problemática, por ello la importancia de su aplicación en la enseñanza a nivel superior, en donde se abordan temáticas por medio de herramientas computacionales, que ofrecen elementos visuales y tangibles como lo son gráficos y procesos interactivos en tiempo real, cuyo interés radica en identificar los parámetros de control para mejorar el proceso o caso de análisis, que se pueden representar por medio de diagramas causales, de flujo y de procesos (Ibarra, 2015).

La educación y la enseñanza identifican problemas lineales y estáticos acorde a lo que se dese obtener con el propósito de facilitar el proceso, sin embargo, es difícil mostrar las problemáticas tal cual se presentan en la vida real; por ello Forrester establece en una de sus publicaciones relacionadas directamente con la parte educativa que: "La mente humana toma imágenes, mapas y relaciones estáticas de una manera maravillosamente efectiva" (Forrester, 1992).

Por otro lado, la tecnología utilizada para el desarrollo de simulaciones representa la realidad con base en modelos matemáticos y lógicos, que traducen el proceso de estudio en un modelo interactivo en donde se logran modificar los parámetros, obteniendo un análisis con alternativas de mejora en función a los resultados al momento de procesar los datos del caso de estudio (Giraldo, 2016)

En este trabajo se aplicará el modelador libre llamado Bizagi, el cual ofrece diversas alternativas de solución a casos de estudio en las diferentes áreas de la ingeniería.

\section{METODOLOGÍA DE DESARROLLO}

En esta sección se describe de manera general un caso para la aplicación de un software denominado Bizagi, el cual ofrece la facilidad de realizar procesos dinámicos obteniendo simulaciones que permiten comprender el proceso en cuestión, por ello la importancia de contar con software que facilite la comprensión de situaciones ingenieriles como lo son procesos productivos e industriales.

Las ventajas que ofrece Bizagi son: reducción de costos, reducción de tiempos en cada proceso, aumento en el control de riesgo, aumentar la cadena de valor, alineación en las áreas de negocios y tecnología, la mejora continua.

Es un programa utilizado en el sector industrial que se logra adaptar a la parte docente con lo que se busca identificar la mejora continua en los procesos de enseñanza aprendizaje de la ingeniería y este software cumple con los requisitos necesarios para la actividad dentro de los procesos industriales en la parte académica.

Para lo cual se tomará como aplicación un caso analizado para un proceso en el estudio del trabajo identificando parámetros de control para la mejora en un proceso teórico que se deriva de un modelo dinámico en donde se controla en tiempo para desarrollar el caso. 
A continuación, se tiene la información del caso en la forma común y posteriormente se traduce con el software para observar la diferencia y lo atractivo que es trabajar con entornos virtuales para mejorar la condición de los métodos de enseñanza-aprendizaje de las ingenierías.

El caso para analizar consiste en el proceso de producción de estrellas para llaveros elaboradas con material MDF, representado por medio de un diagrama de flujo.

El diagrama de flujo se tiene en la figura 1.

Fig.1. Diagrama de flujo convencional del proceso mencionado

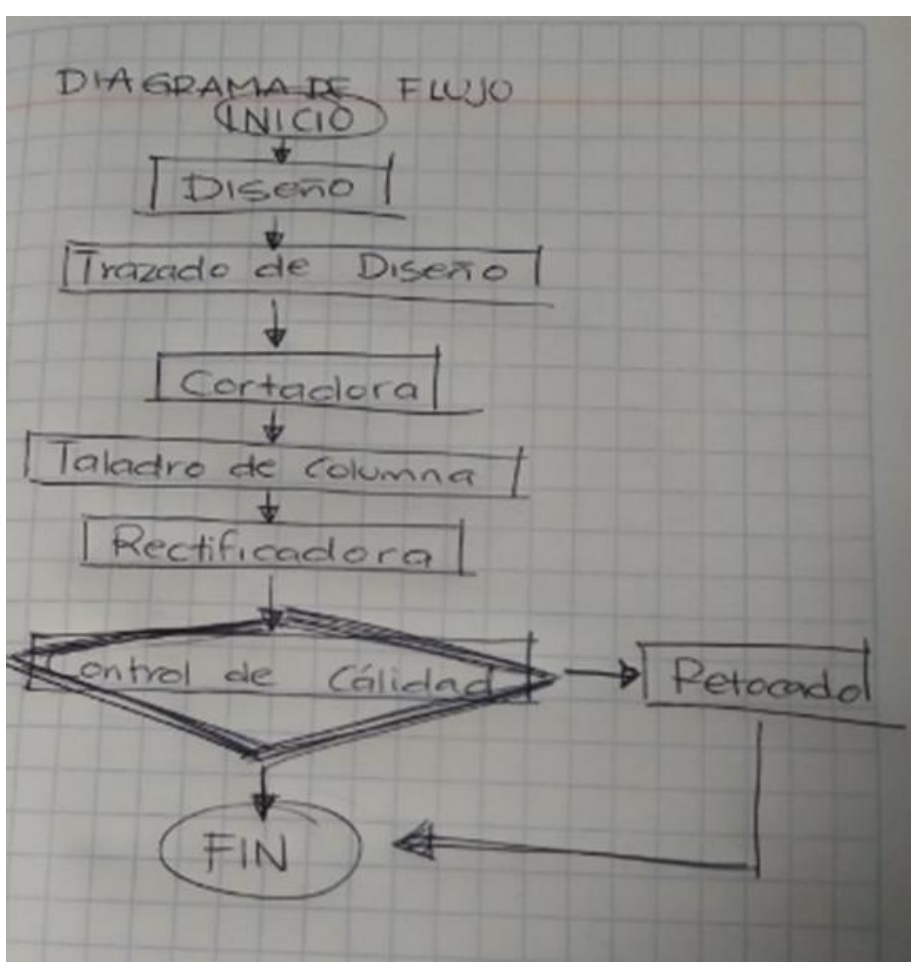

Desde el punto de vista convencional en una clase se realizan los diagramas de flujo de la forma en la cual se describe en la figura 1, la cual ejemplifica el proceso en la producción de llaveros en forma de estrella, para evaluar las etapas en la elaboración del producto.

Ahora el diagrama de flujo con el software se muestra en la figura 2, en donde se logra identificar la manera en la cual se interactúa para determinar los parámetros de control. 
Fig. 2. Diagrama de flujo con el software

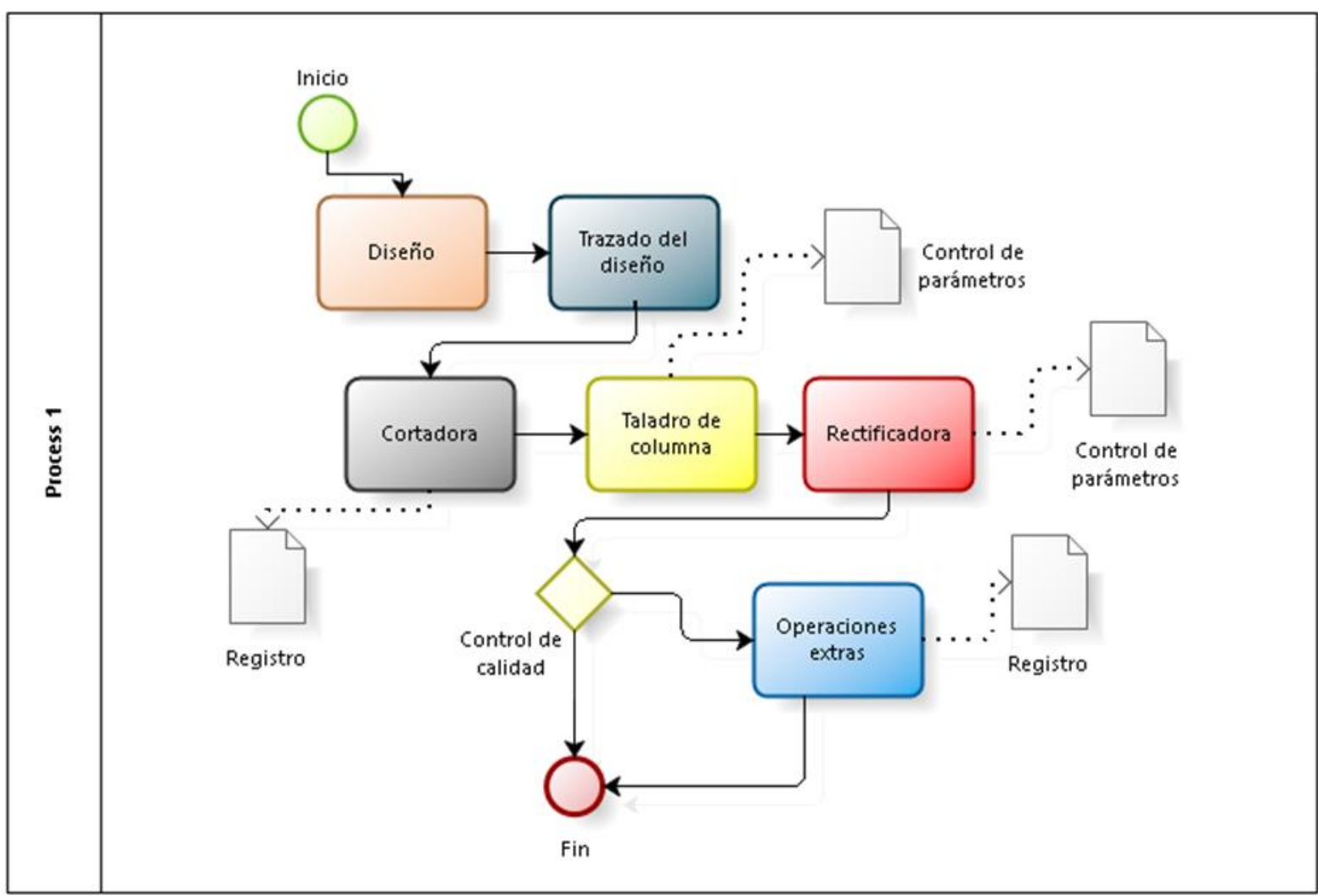

La figura anterior ofrece una visión completa del proceso a través del diagrama de flujo en donde se pueden anexar datos de registro para cada uno de los procesos u operaciones que pueden contener parámetros de control para la mejora continua de cada uno de ellos.

Como parte de las alternativas se logra anexar una gama de imágenes en donde se aprecia de forma general el proceso que se sigue en el momento de realizar los productos y el control de esto mismo, como se aprecia en la figura 3 , con el conjunto de imágenes en el proceso. 
Fig. 3. Imágenes en el proceso para determinar el control en el desarrollo del producto.

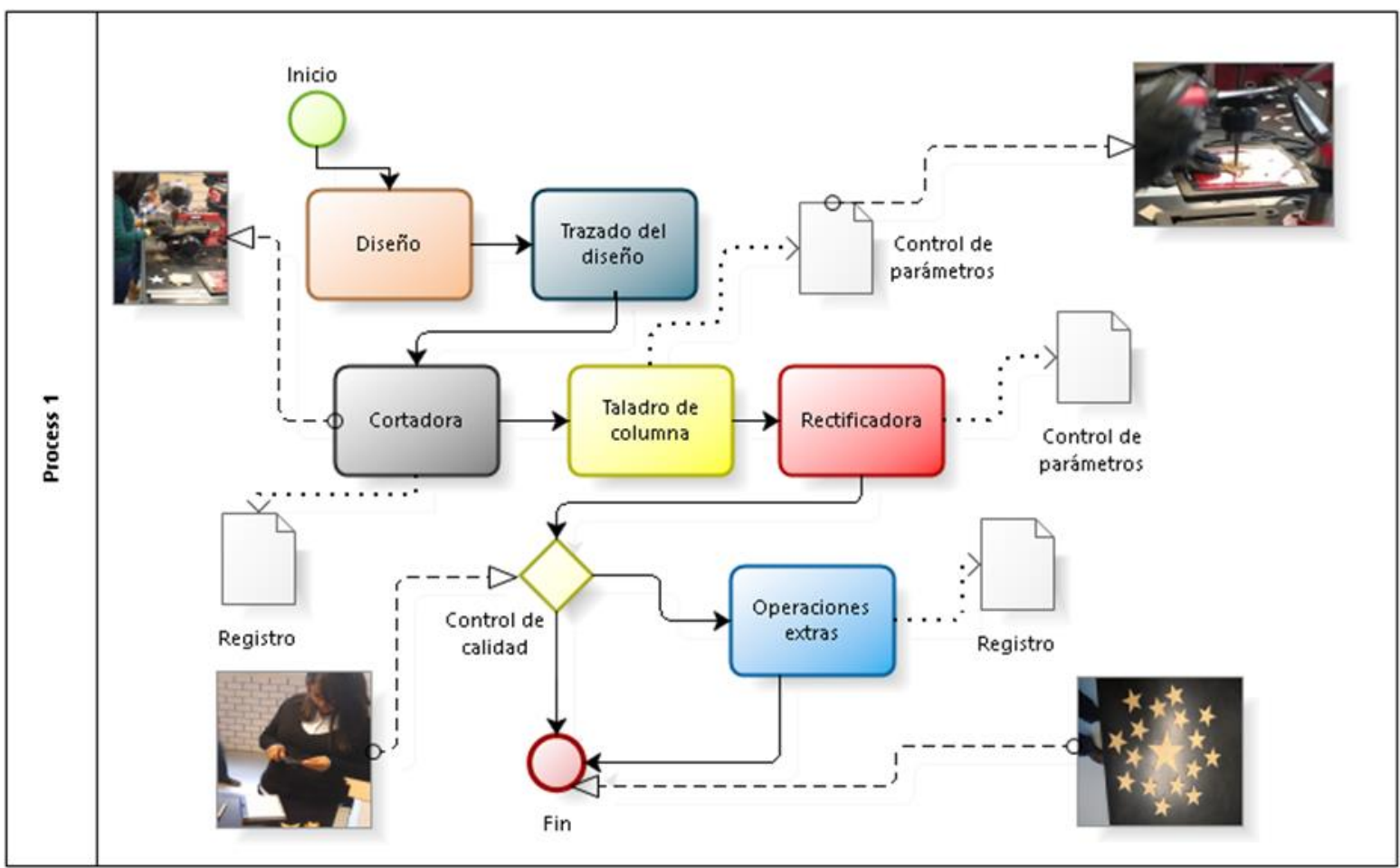

La figura 3 muestra la secuencia del proceso por medio de imágenes para hacer más sencillo el análisis y tangible en su aplicación para determinar los criterios básicos en la elaboración de los productos y el control de la calidad de este mismo.

Posteriormente para todo el proceso se puede seccionar cada una de las operaciones para mantener constantemente la mejora en cada uno de ellos, el primer diagrama es para el caso del diseño, figura 4, que da alternativas de solución para el diseño.

Fig.4. Control del proceso de diseño

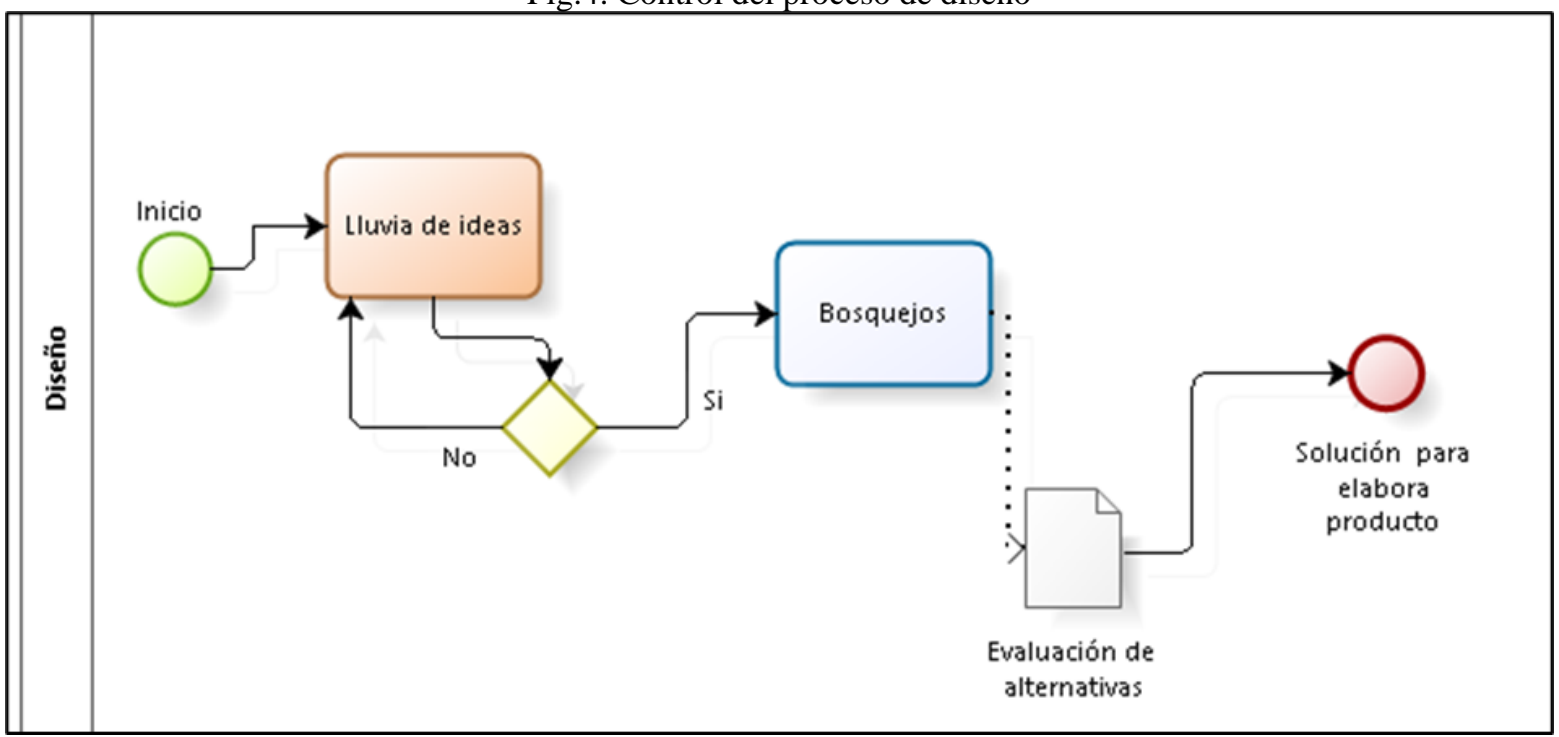


La figura permite identificar en que paso se debe actuar para lograr enriquecer cada una de las alternativas de solución en donde se identifica la sección que se puede manejar como retroalimentación para reiniciar el proceso.

La segunda sección corresponde al trazado del diseño para marcar las figuras en el material MDF, figura 5 .

Fig. 5. Proceso de trazado en MDF

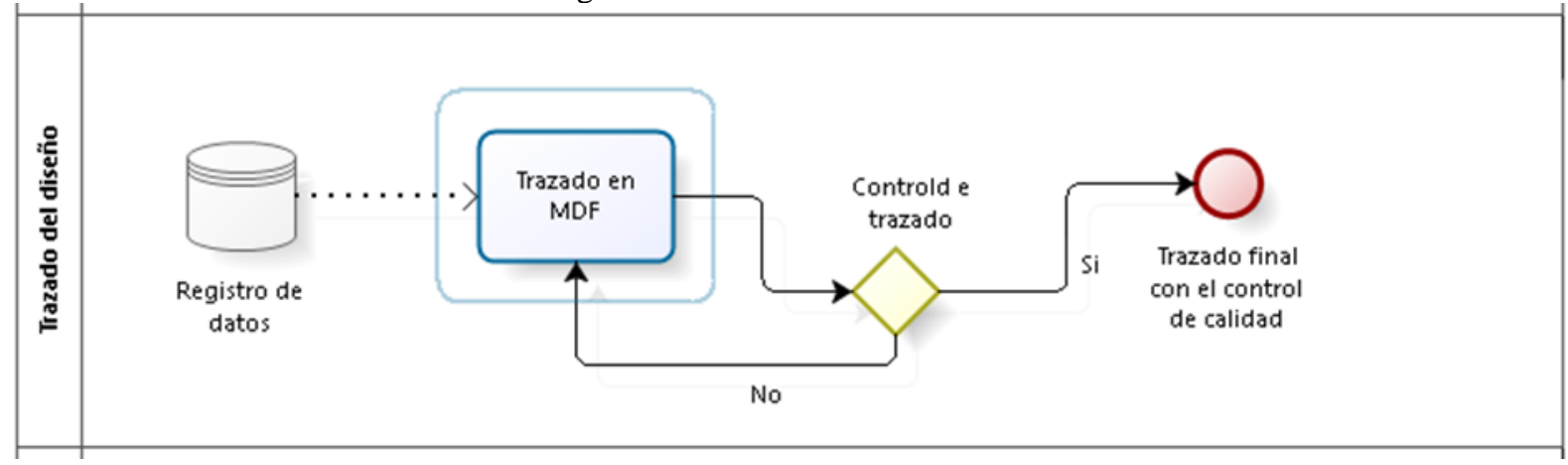

En esta figura se tiene en primer lugar el registro de los datos obtenidos en el proceso anterior, diseño, para lograr el trazado de las figuras en el material para controlar cada uno de los trazos y las herramientas a utilizar.

Como parte de operación se tiene la cortadora y el taladro de columna, en donde se realizan operaciones de corte y barrenado para obtener los productos correspondientes de los llaveros, figura 6.

Fig. 6. Proceso de cortado y barrena.

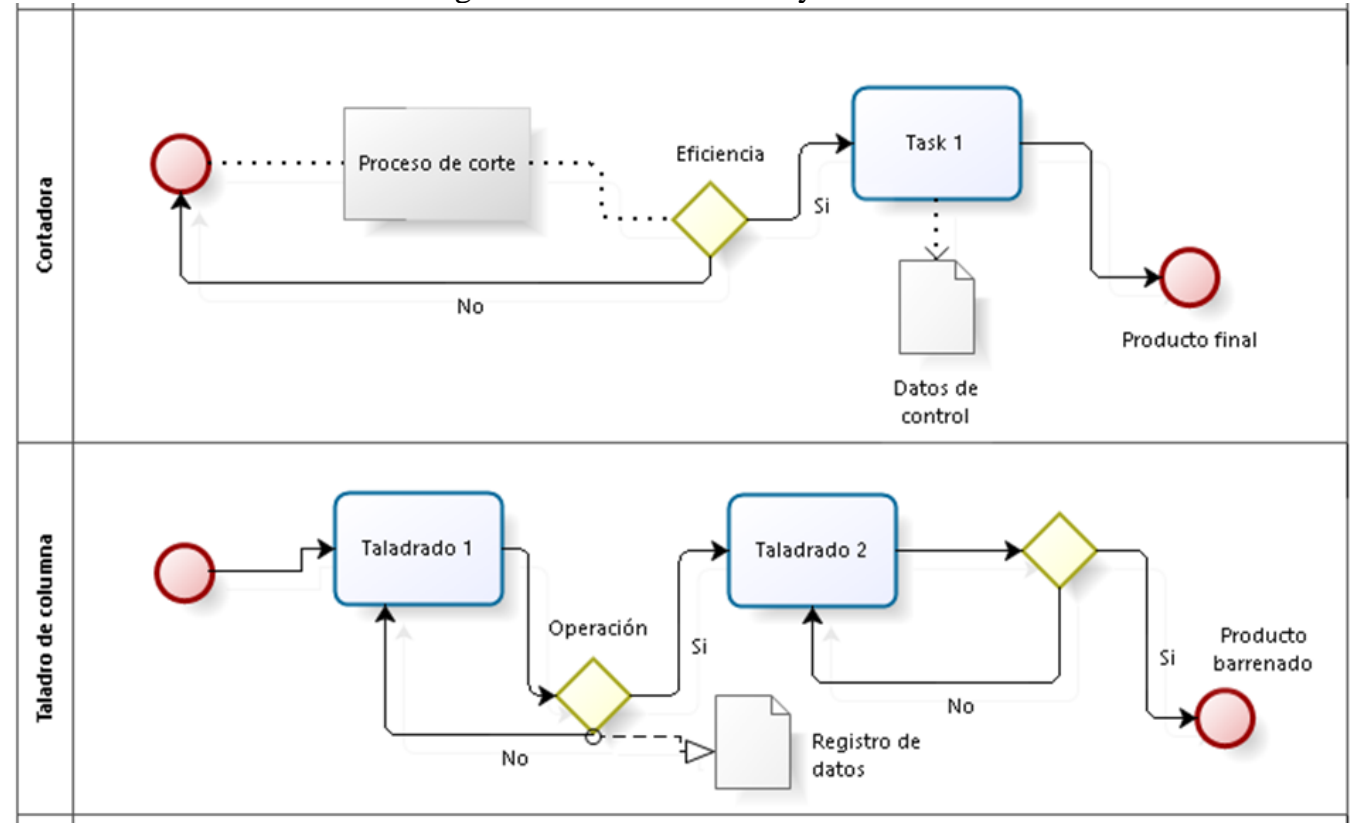


Nuevamente al igual que en las figuras anteriores en la figura 6 se tiene el control de cada proceso para identificar los elementos críticos en cada uno de los procesos.

Como se aprecia en todos y cada uno de los diagramas mostrados en las figuras permiten establecer los controles por medio de un software que mantiene una plataforma amigable al usuario en el momento de aplicar los conceptos en un proceso de estas características.

Los diagramas se plasman de forma personalizada para que el usuario, estudiante, identifique de manera directa la secuencia en cada proceso presente para el producto final.

Existen más vertientes en el software, sin embargo, para este caso se considera necesario considerar los diagramas de flujo como parte de un análisis dinámico de un proceso de producción, sin perder de vista que se pueden obtener gráficas con esta herramienta para contabilizar y evaluar de forma puntual el desarrollo de un proceso, las gráficas permiten visualizar de otra forma el contenido de las operaciones en la producción y los detalles como parte de las variables dependientes e independientes que se logran de los diagramas de flujo y sus alternativas de evaluación.

Por lo que se puede mencionar que la herramienta de Bizagi, se considera completa en el análisis de un proceso para producción con parámetros de control dinámico, en función del tiempo, para la base de datos y/o registro de las operaciones.

\section{RESULTADOS}

Como parte de los resultados en el proceso de producción en el caso de estudio se aprecia en la figura 7 , producto final.

Fig. 7. Producto final en material MDF.

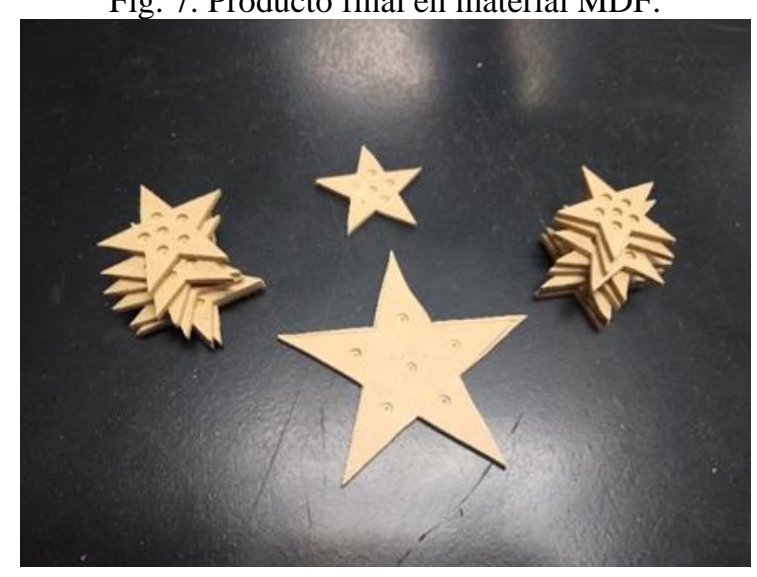


La figura anterior muestra el resultado al llevar acabo de forma ordenada y controlada cada uno de los procesos que se describieron en cada diagrama de flujo de las figuras anteriores, logrando tablas y gráficas de control para el desarrollo del trabajo de producción.

La tabla 1, muestra el control de calidad en la elaboración de los productos en cuanto a la longitud total y la longitud al centro de la pieza.

Tabla 1. Control de calidad producto final

\begin{tabular}{|l|l|l|}
\hline No & Longitud & Centro \\
\hline 1 & 26.25 & 10.07 \\
\hline 2 & 24.36 & 7.74 \\
\hline 3 & 24.73 & 9.24 \\
\hline 4 & 28.12 & 9.24 \\
\hline 5 & 26.42 & 8.89 \\
\hline 6 & 25.17 & 7.65 \\
\hline 7 & 23.56 & 7.85 \\
\hline 8 & 25.93 & 7.88 \\
\hline 9 & 26.63 & 8.13 \\
\hline 10 & 23.6 & 9.04 \\
\hline 11 & 24.48 & 8.68 \\
\hline 12 & 25.1 & 7.68 \\
\hline 13 & 25.94 & 8.72 \\
\hline 14 & 27.14 & 10.47 \\
\hline 15 & 26.03 & 10.02 \\
\hline 16 & 26.6 & 9.23 \\
\hline 17 & 49.55 & 19.83 \\
\hline
\end{tabular}

La gráfica representativa de la tabla anterior se muestra en la figura 8. 
Fig. 8. Gráfico del control de calidad para el producto final

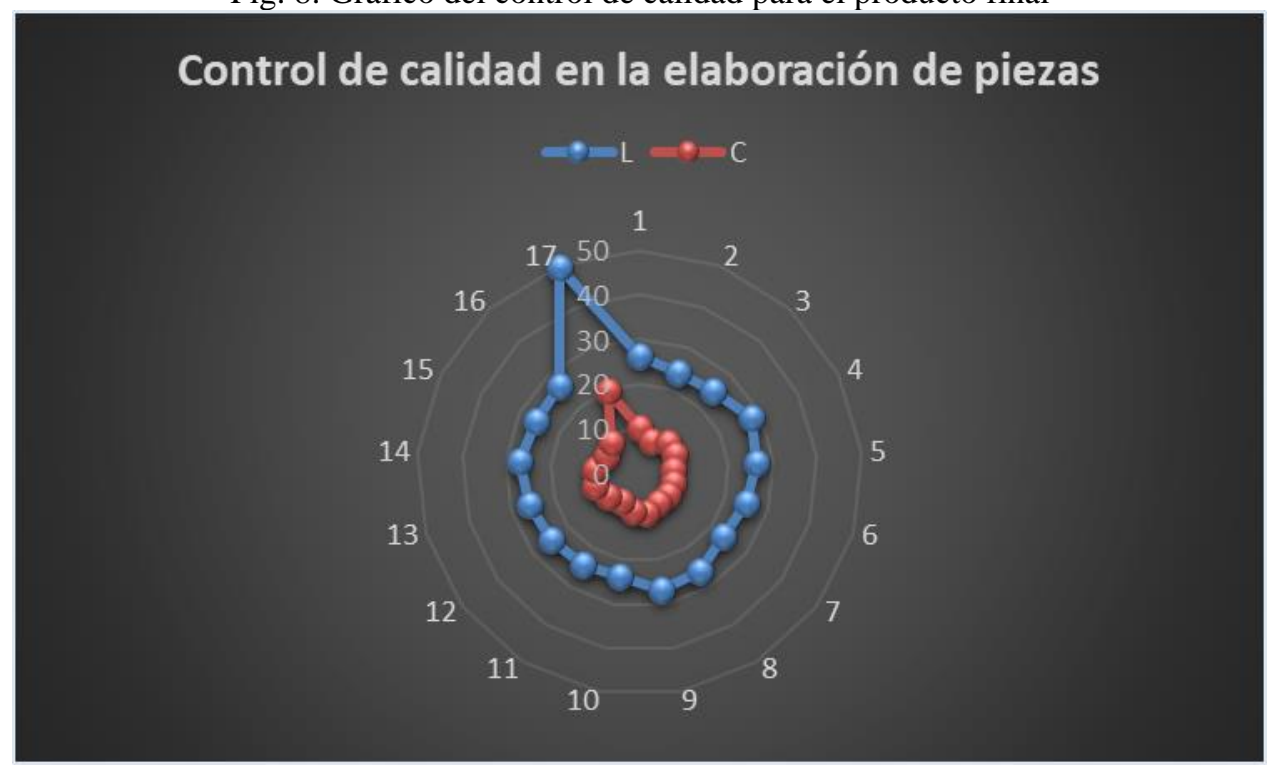

La figura anterior muestra el control de calidad en un gráfico de radar en donde se aprecia que existieron piezas que no cumplieron con el control y fueron la pieza 9 y 17.

Con los datos recabados en los diagramas de flujo se logró desarrollar todo el trabajo de investigación que se muestra.

\section{CONCLUSIONES}

Como parte de las conclusiones se tiene que el uso de herramientas como Bizagi en el desarrollo de procesos dinámicos favorece el aprendizaje, ya que cuenta con sistema amigable para su desarrollo y el aprendizaje mejorando el entorno, parte importante es que los estudiantes quedan satisfechos con los resultados que ofrece el software.

En el caso de estudio algo que reditúa la aplicación de la herramienta es el control del tiempo en cada una de las fases del proceso que se logró disminuir el tiempo de producción a través del control de calidad en cada etapa de este mismo.

Los diagramas de flujo lograron el objetivo de mejorar las condiciones críticas para el control de cada proceso inmerso en sus diversas alternativas de evaluación y el impacto sobre el producto final satisface la condición inicial de cada diagrama de flujo.

Por último, cabe rescatar que se analizarán a detalle los diagramas para formular nuevas tendencias de mejora y lograr implementar las metodologías con estas herramientas para los procesos de enseñanzaaprendizaje en las diversas áreas de la ingeniería, fomentando en todo momento que son herramientas, apoyos de aprendizaje, más no la solución al problema que se presente. 


\section{REFERENCIAS}

Natalia Martínez, Giovanni López, Hugo Andrade, Gerardo Muñoz. (2012). La dinámica de sistemas en un proyecto educativo para la sostenibilidad de un sistema de alerta temprana en salud. X Congreso Latinoamericano de Dinámica de Sistemas, 1, 1-15.

Danny W. Ibarra Vega, Johan Manuel Redondo. (2015). Dinámica de sistemas, una herramienta para la educación ambiental en ingeniería. Revista Luna Azul, 1, 152-164.

Forrester, J. (1992). La Dinámica de Sistemas y el Aprendizaje del Alumno en la educación escolar. Proyecto Educativo Dinámica de Sistemas. Grupo de Dinámica de Sistemas Escuela de Administración Massachusetts Institute of Technology.

Jaime A. Giraldo, Juanita Pinilla. (2016). Simulación de procesos de negocios (BPSIM) como soporte didáctico en el aprendizaje de la gestión de procesos de servicio. Formación Universitaria, 9, 99-108.

Melo-González, Rafael, López-Sánchez, C., Camacho-Gamboa, C., Esparza-Lagunes, A., VillaDomínguez, R. (2012), Simulación dinámica de sistemas, una herramienta para el análisis del desempeño de sistemas y procesos en la industria petrolera: Metodología y resultados. Tecnología, Ciencia, Educación [En línea]: Obtenido en enero de 2019 de la dirección: http://www.redalyc.org/articulo.oa?id=48224413007.

Andrés Beatriz, Sanchis Raquel (2016). Modelado y Simulación de la Cadena de Suministro con AnyLogic. Universidad Politécnica de Valencia.

Fullana B. Carmen, Urquía G. Elena. (2005). Los modelos de simulación: una herramienta multidisciplinar de investigación. Encuentros multidisciplinares, ISSN-e 1139-9325, Vol. 11, N. 32 , 2009, págs. 37-48

Ruiz Romero Magdalena (2017). Estudio comparativo de Herramientas de Simulación. Tesis de Grado. Sevilla. 